ankles were swollen and aching and she was again vomiting repeatedly. The vomitus became blood-stained and by the next morning she was vomiting almost pure blood. She had frequen bouts of abdominal colic with bloody diarrhoea.

On bed rest and clear fluids only she appeared to recover, but 10 days later there was a recurrence of petechiae on the legs, painful swollen ankles, vomiting of blood-stained fluid, and abdominal colic with bloody diarrhoea. She was well again after four days but had recurren episodes of painful and swollen ankles with petechiae on both legs, sometimes accompanied by vomiting, but there was no further blood los from stomach or bowel. These milder episodes recurred every four to eight days, becoming less and less severe. On one of these occasions there was also aching of the elbow joints and petechia on both forearms. Her last day of symptoms was 7 November.

Her urine was consistently free from albumen until 12 November, when there was moderate albuminuria. At this stage she was admitted to hospital under the care of Dr. E. Allibone. After a week on clear fluids and absolute bed rest she felt and looked well, but the albuminuria was unchanged. Prednisone, $15 \mathrm{mg}$. daily, was then commenced, continued at this dose for on month, and then gradually withdrawn over period of three weeks.

After three weeks of prednisone therapy her urine became free of albumen.

She is still Cushingoid but there has been no return of albuminuria to date.

-I am, etc.,

Leeds 7

G. W. LEWIS.

\section{Replacement Arthroplasty}

SIR,-The report of the annual general meeting of the British Association of Physical Medicine (26 March, p. 794) is not accurate in attributing to me the conclusion that replacement arthroplasty of the hip is the treatment of choice in the hip affected by rheumatoid arthritis. In fact excision arthroplasty is probably the treatment of choice at the present time. There is a place for SmithPetersen cup arthroplasty in certain selected cases of secondary osteoarthritis superimposed upon the rheumatoid process, and total replacement with articulated prostheses of the McKee type may become the treatment of choice in the future.

In the knee synovectomy has proved to be valuable in the early stages of the disease before significant joint destruction has occurred. Tibial osteotomy to correct valgus or varus instability is likewise useful in a small number of patients. A combined synovectomy and tibial osteotomy is rarely indicated and it would be unwise to do both procedures in one operation.-I am, etc.,

$$
\begin{aligned}
& \text { Guy's Hospital, } \\
& \text { London S.E.1. }
\end{aligned}
$$

\section{Complication of Contact Lenses}

SIR,-In refreshing contrast to the alarming headlines in national newspapers reporting the recent analysis of 50,000 contactlens-wearing patients in the U.S.A., your leader on complications of contact lenses ( 26 March, p. 751) deserves high commendation for its objectivity.

It is, of course, true that a contact lens combining all of the desirable qualities you mention is not yet available, though presentday lenses, when skilfully fitted, periodically checked, and hygienically cared for are capable of excellent performance over many years.

To your list of possible causes of complications I would add neglect by the patient of the practitioner's advice to attend for regular aftercare, for the absence of untoward symptoms tends to create a degree of confidence beyond reasonable justification. I welcome, therefore, your emphasis on the importance of aftercare and of strict attention to hygiene in lens handling and maintenance.

We recommend that the fitting of lenses should be preceded by reaction tests to determine the suitability of the eyes for this form of correction, and that the initial fitting should be followed by frequent inspection of the eyes and lenses for one year. Subsequently, an annual re-examination is advised.

Your suggestion that the development of contact-lens technique calls for cooperation between the medical and optical professions is timely and will be endorsed by all engaged in this work. The International Society of Contact Lens Specialists, which includes in its membership ophthalmologists, optometrists, and opticians, was founded some 15 years ago for the advancement of contact-lens technique and research. I intend to draw the attention of our members to your valuable article at our forthcoming congress in Mexico City, 20-24 April.-I am, etc.,

$$
\begin{aligned}
& \text { FRANK DICKINSON. } \\
& \text { Secretary, } \\
& \text { International Society of } \\
& \text { Contact Lens Specialists. }
\end{aligned}
$$

St. Annes-on-Sea,

$$
\text { Lancs. }
$$

\section{Follow-up}

SIR,-The job of a consultant is, by tradition, to be consulted by a practitioner about a patient. Except in a few specialties, and for purposes of research, it is not necessary for a consultant to do extensive personal follow-up. This is the job of the family doctor, by tradition, who can always seek the further opinion of a consultant when necessary.

Follow-up by or repeated visits to a consultant are sometimes necessary nowadays only in obstetrics, psychiatry, and in the " newer" specialties such as cardiac surgery. If consultants were freed of the chores of which Mr. Rowntree complains they would have more time for new consultations and so shorten the outpatient waiting-lists.

Finally, there is no reason why any doctor should have to do routine work on Saturdays when the vast majority of our patients only work a five-day week.-I am, etc.,

$$
\text { London N.W.11. }
$$

D. S. NACHSHEN.

SiR,-Your correspondent Mr. T. Rowntree (19 March, p. 738) can be assured that he will get no angry stream of abuse from at least one general practitioner. For years I have fought an uphill battle to retain my patients under my own care and to use the consultant service as it should be used. My experience after 18 years in general practice is that consultants in the main are not the least bit interested in the early return of patients to their own doctor's care.

It is accepted without question by the Government and all medical authorities that the consultant establishment is seriously undermanned. My own experience of the attitude of consultants to follow-up cases leaves me to seriously doubt that this is so.I am, etc.,

London $\mathbb{W} .13$.

JoHN H. Swan.

\section{Foetus in Hernia}

SIR,-I should like to relate to your readers a case of an unusual obstetric presentation.

The woman in question was gravida five, with the first three pregnancies normal deliveries and alive, but the fourth was a normal delivery with stillbirth.

She presented at full term with the gravid uterus herniated through a large rectus abdominis deficiency and so anteflexed that in the erect position the fundus lay at the level of the knees. The head was presenting but not engaged. The uterus was reducible to the vertical position.

She was offered operative treatment in which the child could be removed by caesarean section and repair of the rectus deficiency effected. This she refused on the grounds that she had attained normal delivery under these circumstances before, and she could do it again. We were doubtful about her ability to do this but had no alternative.

When she went into labour she got one of her relatives to hold the uterus up in a vertical position and delivered without any difficulty.

She was offered a repair of the hernia subsequently, but again refused, saying, "This is how God made me, and this is how I am going to stay." - I am, etc.,

Kabale, Uganda.

R. A. Jones.

\section{Misdiagnosis of Dysphasia}

SIR,-I was most interested in Dr. N. Berlyne's letter on this subject (12 February, p. 421), agreeing with him that where it is a question of urgent or active general medical or neurological treatment most psychiatric hospitals would probably not be very suitable places ; but admission there later, in the residual or longer term therapeutic stage, is neither inappropriate nor uncommon. ${ }^{2}$ Not only are long-term neurological and general medical beds probably in relatively short supply, but the latter would be less suitable than a psychiatric hospital on grounds of medical and nursing psychological understanding, from which such patients clearly benefit.

There is another aspect of "misdiagnosis" in dysphasia, however, to which attention should also be drawn, involving the cerebral pathophysiology of speech function. Thus Dr. J. D. Spillane's patient ${ }^{2}$ with left temporal lobe abscess showed left frontotemporal brief dysrhythmia when unable to speak, but posterior temporal dysrhythmia when unable to understand, the E.E.G. in fact proving superior to morbid anatomy in localization of dysfunction.

I have made a somewhat similar observation on a patient nursed in this psychiatric hospital, whose clinical details have been described elsewhere." t His death here (9 January 1965) due to cerebral haemorrhage, four years after the only other vascular 